\title{
Téoros
}

Revue de recherche en tourisme

\section{Tourisme, santé et bien-être}

\section{Luce Proulx}

Volume 24, numéro 3, automne 2005

Tourisme et santé, quelques perspectives

URI : https://id.erudit.org/iderudit/1071074ar

DOI : https://doi.org/10.7202/1071074ar

Aller au sommaire du numéro

\section{Éditeur(s)}

Université du Québec à Montréal

\section{ISSN}

0712-8657 (imprimé)

1923-2705 (numérique)

Découvrir la revue

Citer cet article

Proulx, L. (2005). Tourisme, santé et bien-être. Téoros, 24(3), 5-11.

https://doi.org/10.7202/1071074ar d'utilisation que vous pouvez consulter en ligne.

https://apropos.erudit.org/fr/usagers/politique-dutilisation/ 


\section{Tourisme, santé et bien-être}

\section{Luce Proulx}

Le tourisme est trop souvent vu simplement à partir de sa finalité économique, c'est-àdire l'industrie touristique. Pourtant, il recèle des dimensions humaines et sociales importantes. D'ailleurs, à ses origines, c'est par ses bienfaits sur les individus que l'on justifiait les déplacements qu'il suppose.

C'est principalement au siècle des Lumières (XVIII') que le tourisme fait son apparition en Angleterre, pays qui subissait depuis un moment déjà des changements sociaux importants dans ses classes dirigeantes. L'aristocratie est en voie de devenir une classe sans pouvoir et l'idée du voyage émerge pour s'occuper, pour fuir une situation sociale inconfortable. Mais partir sans motif valable est, à l'époque, socialement inacceptable. C'est dans un tel contexte que le prétexte de la santé prend son sens. Les sources thermales deviennent les premiers lieux de rassemblement de ces " touristes ". Notons, cependant, que le thermalisme était déjà connu depuis l'Antiquité, époque où les Romains en avaient découvert les bienfaits.

Les aristocrates se regroupent donc dans ces lieux de santé, "contemporanisés ", à des fins thérapeutiques et curatives, mais aussi pour recréer leur société et rechercher du plaisir à travers diverses activités mondaines (bals, repas gastronomiques, sport, casino, etc.). Des healthy places qui sont autant des pleasure places et des lieux de distinction (Boyer, 1996); des espaces-temps différents de la vie ordinaire basés sur un univers ludique où la rencontre et le divertissement se vivent dans un état de surstimulation (Laplante, 1997). Bath en est le berceau. II devient "un lieu où on se soucie moins des bains, ni des eaux à prendre, plutôt qu'un lieu pour se divertir en bonne compagnie » (Boyer,

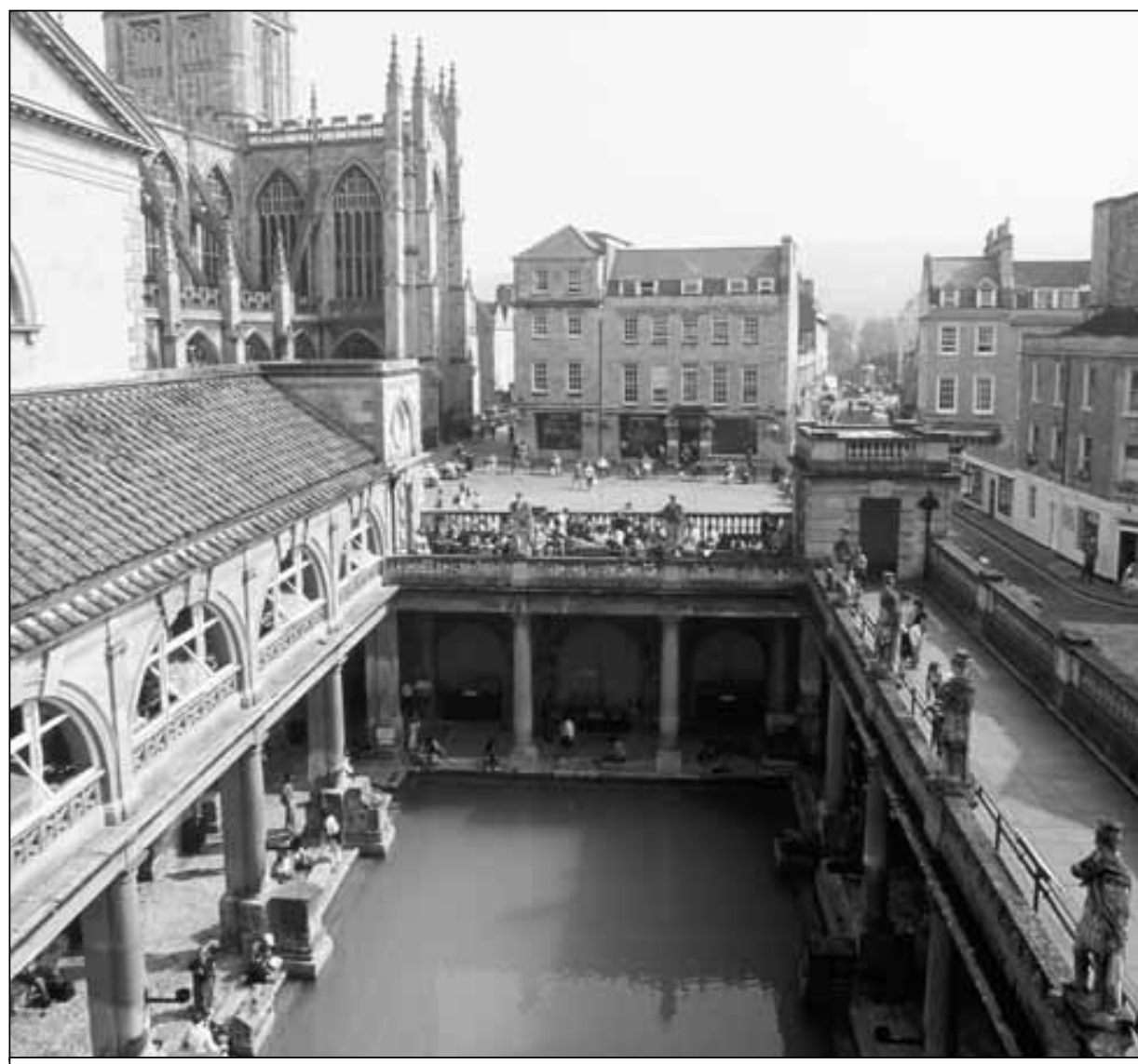

Bain romain dans la ville de Bath.

Photo : Bath Tourism

1996 : 37). À Bath, suivront d'autres villes d'eau toujours plus à la mode.

Au discours thérapeutique se conjugue subséquemment le discours hygiéniste $\left(X V I I{ }^{e}\right.$ et XIXe siècles). Les voyages permettent de "guérir l'âme ou le corps", mais aussi de " changer d'air ». II y a ainsi des lieux comme Nice dans le Midi de la France destinés aux hivernants à la santé délicate et aux malades qui cherchent le calme. Suit l'attrait de la campagne $^{1}$, cette belle nature qui répond à un besoin « d'air pur » et de " retour à la nature » pour les citadins, les plus riches évidemment. Effectivement, la ville devient malsaine: lieu de perdition, de corruption, d'épidémies, d'insalubrité, etc. C'est la naissance du phénomène de la villegiatura. On voit s'installer des maisons de campagne, pour ne pas dire des domaines ou des châteaux, pour le «bonheur $^{2} \gg$ que procure la campagne, voire la nature. Effectivement, cette valeur du bien-être s'impose au Romantisme comme un droit aux plaisirs de la vie. 
Pouvoir médicinal de l'eau salée et bienfaits thérapeutiques de l'air de la campagne, le prétexte de la santé domine donc initialement toutes préoccupations «touristiques» en rendant vertueux les déplacements des premiers touristes, tandis que l'ère du Romantisme viendra justifier le "plaisir» comme motif du voyage. Mais c'est avec I'histoire du temps libre et celle du loisir que l'on doit saisir le tourisme d'aujourd'hui. Le loisir, ayant été d'abord réservé à l'élite socioéconomique, se démocratisera progressivement dans la première moitié du $X X^{e}$ siècle avec la reconnaissance formelle du droit aux congés payés pour tous les travailleurs et ce, dans plusieurs pays occidentaux. Une démocratisation qui trouve aussi ses racines auprès d'arguments salutaires pour la santé et le bien-être des individus, voire des travailleurs. Effectivement, ce temps «hors travail», ou temps libre, relativement long est vu comme bénéfique à la reproduction de la force de travail et à l'efficacité des travailleurs grâce au repos et au ressourcement qu'il procure. Le repos a laissé sa place aux loisirs, ces activités comprises dans le temps libre dégagé de toutes obligations personnelles, familiales, sociales et civiques, librement choisies par les individus en fonction de leurs goûts, leurs habiletés, leurs aspirations ou leurs ambitions (CQL, 2003). Aujourd'hui, le loisir n'est plus exclusivement l'antidote au travail. II est reconnu comme un droit pour tous $^{3}$ (même si son accès est inégalement réparti dans la population), notamment pour ses effets thérapeutiques, éducatifs, sociaux et moraux.

Même si une offre de services touristiques exclusivement axée sur la santé et le bien-être existe encore aujourd'hui avec le thermalisme ou la thalassothérapie, c'est à partir de la conception du tourisme comme activité de loisir que nous nous proposons d'analyser son impact sur les individus et les collectivités. S'impose d'abord une meilleure compréhension du tourisme comme composante du loisir ainsi que du sens à donner au « bien-être ». Considérant que les recherches portant sur l'apport du tourisme au bien-être individuel et collectif sont peu nombreuses, un détour par la compréhension du loisir comme déterminant du bien-être s'avère nécessaire. Nous reprendrons ensuite ces éléments d'information selon les spécificités du tourisme.

\section{Quelques précisions}

\section{Tourisme}

Le tourisme est une activité de loisir car il se situe d'abord dans le «temps libre» dégagé de toutes obligations, voire le temps de loisir le plus long de l'année, celui des vacances; il réfère à une activité «librement choisie» pour le simple plaisir qu'elle procure; il est " désintéressé », c'est-à-dire gratuit et non lié à la recherche de prestige ou de revenus ; il est "hédoniste ", car il sous-entend la recherche de plaisir et de satisfaction personnelle ; finalement, appartenant à l'individu et contribuant à son développement, il est «personnel».

Poursuivons en ajoutant que le tourisme est une activité de loisir consacrée au voyage, soit un déplacement vers l'Ailleurs, d'où probablement l'expression courante «Partir en vacances ». II appartient à l'univers du loisir "dans la mesure où tourisme et loisir sont des réalités comprises comme appartenant à un univers culturel particulier, celui de la fête et du jeu » (Laplante, 1997 : 41). Ce voyage touristique se réalise donc en dehors de la vie ordinaire et des contraintes quotidiennes, dans une sorte d'état second de défoulement, de libération et de prodigalité. En plus, le jeu a comme fonction sociale la rencontre et la cohésion sociale car il suppose la compagnie des autres.
Il importe, à notre avis, de distinguer ici l'activité vacancière de l'activité touristique. Sans aucun doute, les vacances, comme le tourisme, sont un moment de détente et de ressourcement. Cependant, l'activité touristique est une sous-catégorie de l'activité vacancière et implique une mutation de «l'Homme en vacances » en «Touriste » et pose comme condition une rupture avec la vie quotidienne que rend possible le déplacement. On distingue deux variantes de l'activité touristique: 1) axée sur le repos et comportant un transfert de sédentarité dans un autre lieu pour répondre à un besoin de bonheur sans contraintes (villégiature, retraite, cure) ; 2) axée sur la découverte et animée par le goût de la mobilité. Deux types de tourisme qui apportent leur lot de bienfaits sur les individus.

\section{Santé et bien-être}

L'Organisation mondiale de la santé (OMS) définit la santé comme "un état de complet bien-être physique, mental et social, [qui] ne consiste pas seulement en une absence de maladie ou d'infirmité " 4 . Le terme «bien-être » réfère, selon différentes définitions de dictionnaires ${ }^{5}$, à une sensation de plaisir et de bonheur qui résulte de la satisfaction de ses besoins et l'absence d'inquiétudes. II dépend alors d'un équilibre et d'une harmonie, sur une base quotidienne, entre tous les besoins - physiques,

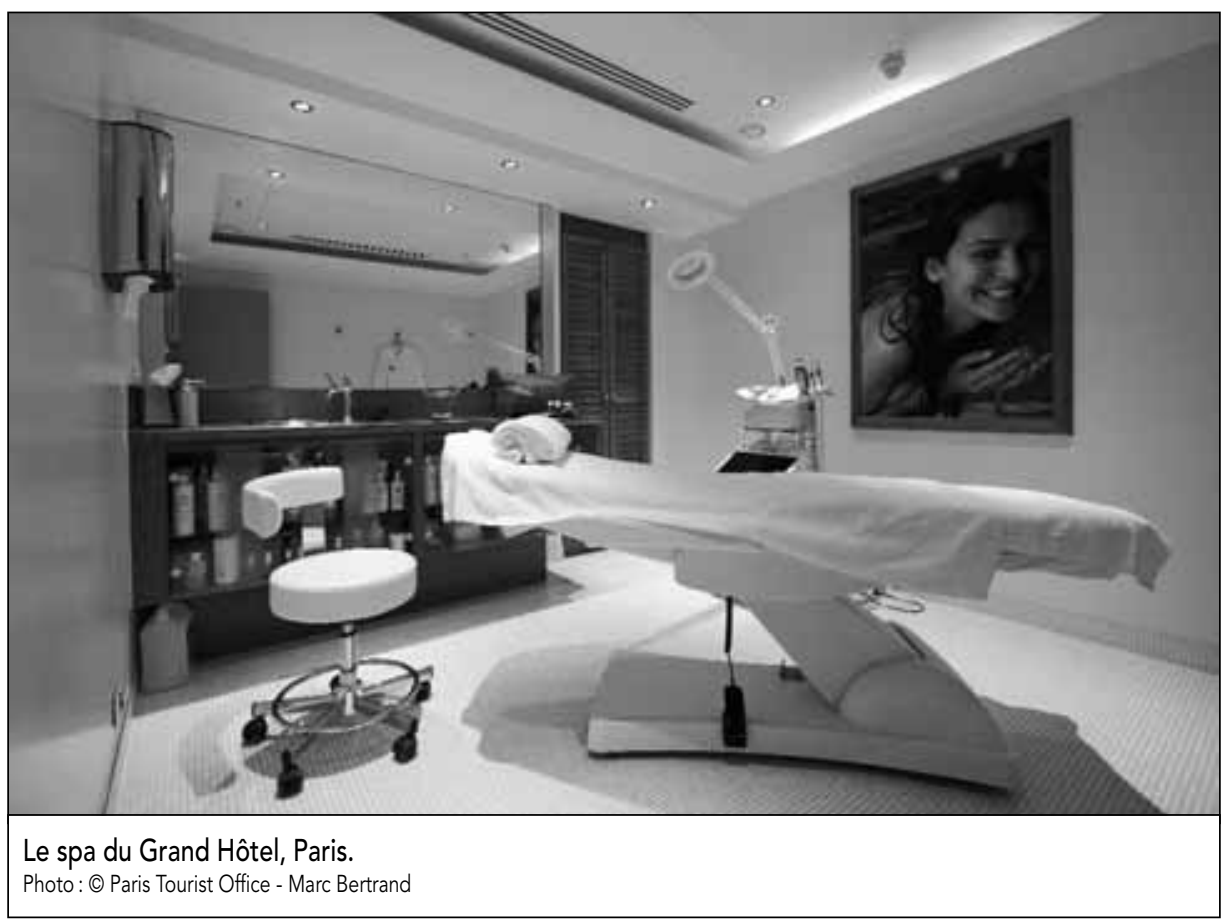


mentaux et sociaux, auxquels nous ajouterons les besoins émotionnels et spirituels - de l'individu. Cette dimension du bien-être introduit donc des éléments, interdépendants, de différents domaines de la vie courante: l'exercice physique, les conditions de stress, mais aussi l'alimentation, le logement et les caractéristiques du milieu environnant, l'estime de soi, les contacts sociaux... ainsi que les loisirs et les départs annuels en vacances.

Pour une vie équilibrée et en santé, tous conviendront qu'il est nécessaire d'être à la fois en bonne forme physique et mentale et de pouvoir satisfaire ses besoins. Jef Breda (1998) introduit à la notion de bien-être trois autres dimensions que nous trouvons intéressantes, parce qu'elles rejoignent la question «sociale " de la définition de l'OMS. Le bien-être signifie que chaque individu peut 1) participer à la vie sociale, 2) s'intégrer dans la société moderne, 3) et développer une personnalité autonome, voire s'émanciper. II propose la définition suivante:

Le bien-être est la mesure dans laquelle non seulement les besoins "primaires" comme l'alimentation, l'habillement, la santé, le logement et un climat social positif sont satisfaits, mais aussi la mesure dans laquelle l'individu participe à la vie sociale et prend part à sa culture et ses valeurs, et où il peut s'épanouir pour développer une personnalité faisant face aux contraintes sociales (1998: 2).

Malgré que le «bien-être » commence par soi, il n'est pas que de seule préoccupation individuelle. Effectivement, cette notion a aussi une dimension collective dans la mesure où la collectivité doit répondre de façon satisfaisante aux besoins de sa population, notamment en agissant sur la pauvreté, l'intégration des groupes marginaux, l'emploi..., voire réduire les inégalités et assurer une meilleure qualité de vie. La participation sociale des individus ou des groupes d'individus, leur intégration à la vie collective (surtout des groupes marginaux) et leur émancipation grâce au développement d'un sentiment d'appartenance sont trois stratégies d'action préconisées dans une approche renouvelée de développement social (Conseil de la santé et du bien-être, 1998), stratégies qui ressemblent étroitement à celles que Breda propose pour assurer le bien-être des individus.

\section{Le loisir comme déterminant du bien-être}

Sue (1980) reconnaît au loisir différentes fonctions de nature psychosociologique et sociale. Sur le plan psychosociologique, le loisir est essentiel à l'équilibre psychologique de l'individu par trois fonctions principales : détente, divertissement et développement. Ses fonctions sociales les plus significatives sont de socialisation, symbolique (signe d'affirmation personnelle et d'appartenance) et thérapeutique. Résultent de ces fonctions plusieurs bienfaits personnels et sociaux (c'est-à-dire qui favorisent la santé des familles, des quartiers et des groupes d'intérêt) que l'Association canadienne des loisirs/parcs détaille sous huit principaux messages de résultats (1998). Brièvement,

1. Les loisirs et la vie active sont des facteurs déterminants de l'état de santé des individus. Ils aident les gens à vivre plus longtemps en améliorant leur santé générale et leur mieux-être, notamment en réduisant les risques de nombreuses maladies. Les loisirs sont des éléments essentiels à la qualité de vie par leur action préventive; ce sont des outils thérapeutiques éprouvés qui aident à rétablir les capacités et les aptitudes physiques, mentales et sociales des individus.

2. Les loisirs représentent la clé du développement équilibré de l'être humain. Ils permettent l'amélioration des aptitudes motrices et sociales ainsi que la créativité, les facultés intellectuelles et plusieurs autres aptitudes utiles dans la vie. Ce sont des occasions d'apprentissage exceptionnelles qui contribuent de façon importante à faire découvrir sa spiritualité. Les loisirs sont de puissants leviers d'affirmation de soi, de conception de soi et d'identité, spécialement parce qu'ils sont librement choisis.

3. Les parcs et les loisirs sont essentiels à la qualité de vie. Ils améliorent positivement l'estime et l'image de soi, des composantes essentielles pour une vie personnelle de qualité. Également, ils améliorent le taux de satisfaction face à la vie et à la qualité de vie perçue des individus, des familles et des communautés et favorisent l'épanouissement, l'acquisition d'aptitudes qui durent toute la vie et l'autonomie des personnes qui ont un handicap. Ils peuvent être des outils pertinents dans un contexte social fragilisé en raison, par exemple, de l'appauvrissement, l'exclusion, la précarisation familiale, etc.

4. Les loisirs réduisent les comportements antisociaux et autodestructeurs. Ils représentent un antidote au tabagisme, à l'abus de drogues et d'alcool, au suicide et à la dépression. Ils réduisent les activités sociales négatives comme la criminalité et le racisme (en améliorant les connaissances sur les différentes cultures). Ils contribuent également à briser l'isolement, la solitude et l'aliénation mentale.

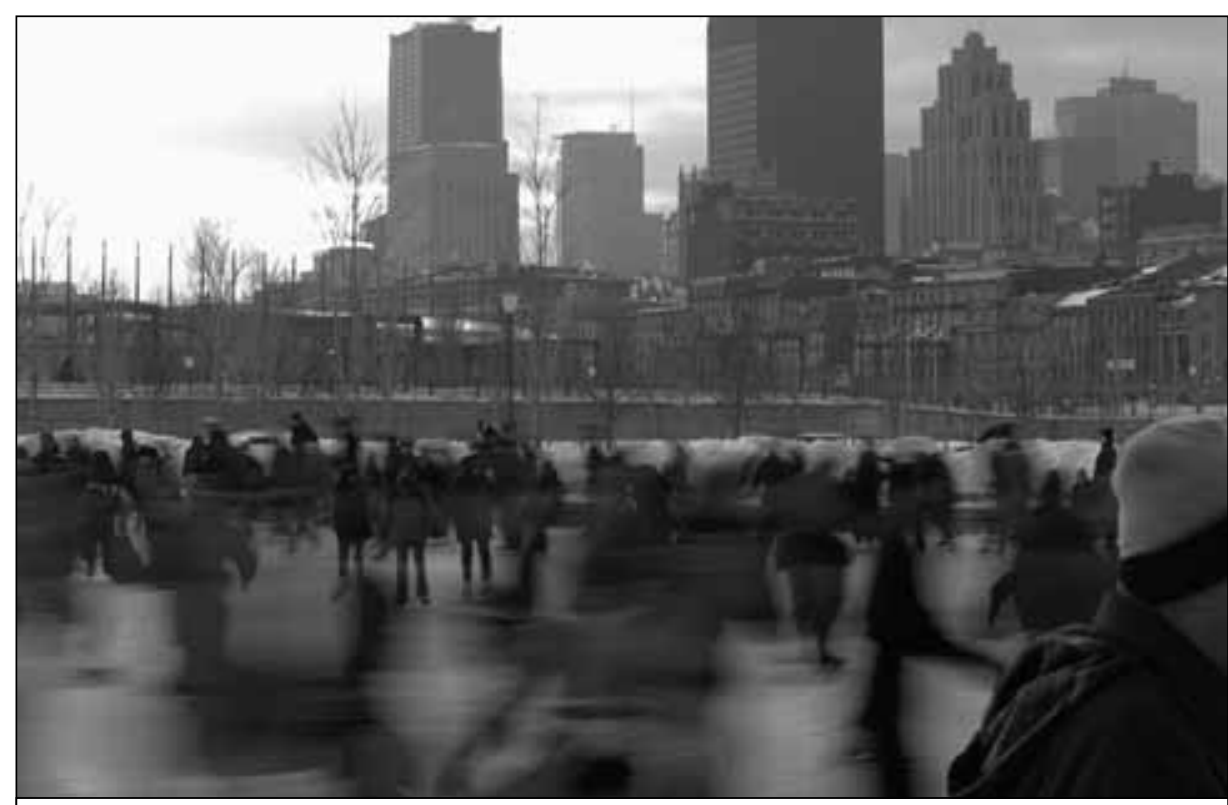

Scéance de patinage dans le Vieux-Montréal.

Photo: Tourisme Montréal/Daniel Choinière 
5. Les parcs et les loisirs contribuent à bâtir des familles fortes et des communautés en santé. Dans un contexte de loisir, les familles jouent et demeurent ensemble et les enfants restent liés. Les loisirs contribuent à 'enrichissement social en offrant des occasions de développement et de formation dont les nouvelles acquisitions peuvent, en retour, être mises au profit de la communauté de diverses façons. Ils inculquent des aptitudes sociales, favorisent la participation à la vie communautaire et collaborent à la cohésion sociale, voire à la création du tissu social, et ils améliorent la fierté communautaire en faisant connaître le milieu, son histoire et son environnement, voire son identité.

6. Les loisirs réduisent les coûts des services de santé, des services sociaux et des services de police/justice.

7. Les parcs et les loisirs sont d'importantes sources d'activités économiques dans nos collectivités. Des études ont démontré que les loisirs améliorent effectivement le rendement au travail et la productivité des travailleurs et réduisent l'absentéisme, le roulement de personnel et le nombre d'accidents de travail. En plus, par la qualité de vie qu'ils supposent, ils attirent des entreprises qui facilitent à leur tour le développement économique du milieu et la création d'emplois (nécessaire à la satisfaction des besoins des individus).

8. Les parcs, les espaces ouverts et les espaces naturels sont essentiels à la survie écologique. En plus de protéger la biodiversité, ils améliorent la qualité de l'air, offrent une excellente approche à l'éducation environnementale qui est à la base de l'autonomie à long terme et représentent un des meilleurs moyens d'exprimer la spiritualité de la terre et d'encourager l'écocivisme.

Sans qu'ils satisfassent l'ensemble des besoins de l'Homme, on constate, dans ces messages, que les loisirs contribuent néanmoins à la bonne forme physique et mentale et au bien-être social et spirituel des individus et des collectivités en agissant sur la participation, l'intégration, l'émancipation et l'affirmation personnelle et communautaire. Autrement dit, la pratique d'activités de loisir (sportive, récréative ou touristique) et la mise en place d'une offre de loisirs par les collectivités influent sur la qualité de vie. Mais le loisir n'est pas qu'« activité ». Le Conseil québécois du loisir (CQL, 2000) le définit aussi comme un mode de relations entre les gens, lequel permet la création de liens sociaux entre les individus et la dynamisation de l'action collective. Ces relations sociales en font un outil de prévention important en matière de santé (car les liens sociaux sont reconnus pour exercer une influence positive sur la santé) et pour contrer l'appauvrissement, l'isolement et l'exclusion. Effectivement, le milieu du loisir - spécialement les organismes de la société civile en loisir - est sensible à l'épanouissement des groupes vulnérables ou fragilisés (jeunes, familles ou personnes aux prises avec un handicap, familles à revenus modestes, etc.).

II ne faut donc pas seulement voir le loisir qu'à partir de sa dimension récréative, mais aussi comme un moyen de développement social. Comme le mentionne Lucie Fréchette (2000: 23), il y a une valeur sociale du loisir qui s'ajoute à sa valeur récréative, laquelle "se traduit par une perception du loisir comme instrument favorisant le développement de la personne, de la famille et du quartier ».

\section{Loisir touristique et bien-être}

L'apport du tourisme au bien-être des individus, des familles et des collectivités est multiple et varie selon la nature du voyage et les types d'activités pratiquées. II peut être distingué selon qu'il agit sur le plan individuel et familial ou collectif.

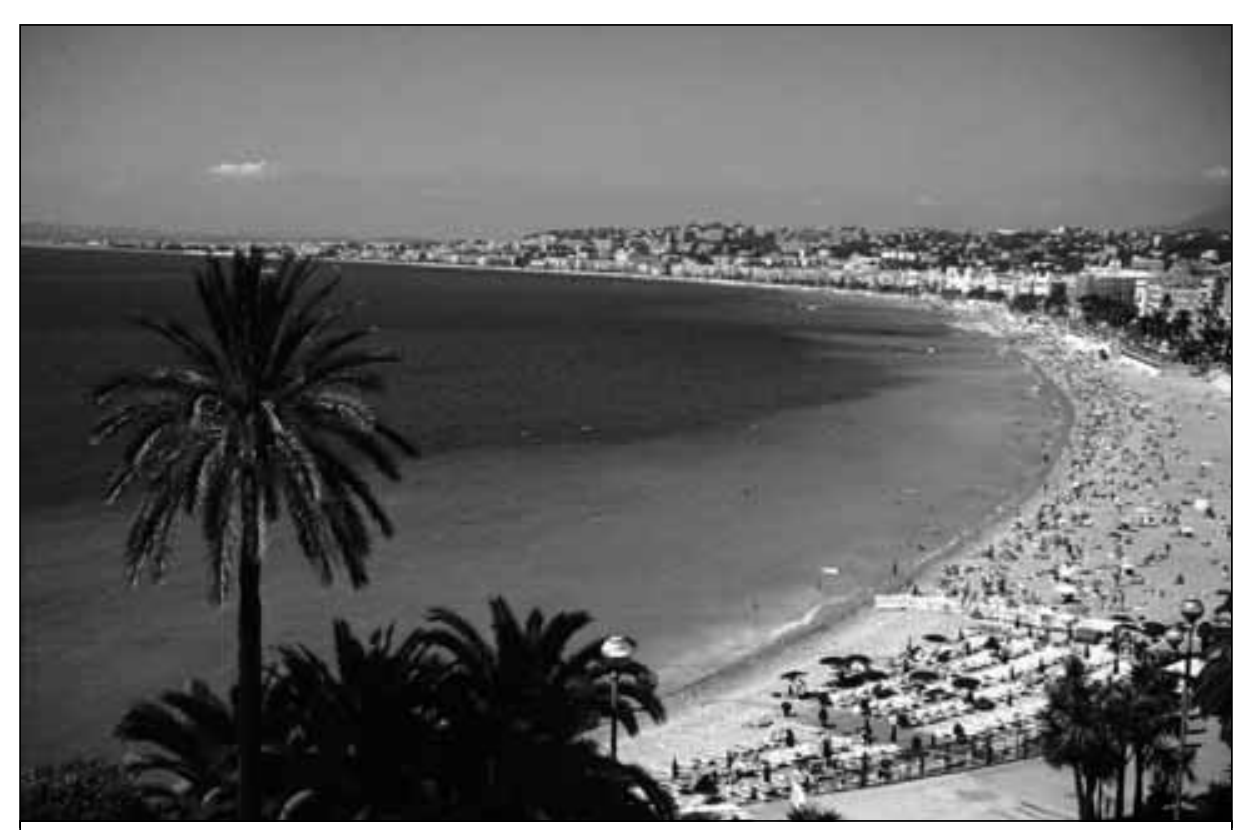

Quai des États-Unis sur la plage de Nice, France.

Photo: Office du Tourisme et des Congrès de Nice

\section{Le plan individuel et familial}

Pour examiner les effets positifs du loisir touristique sans tomber dans une énumération vide de sens, nous privilégions une analyse à partir de quatre spécificités proposées par le Groupe confluences (1996) :

- le voyage touristique réalise d'abord une "rupture » avec le milieu de vie habituel ;

- le déplacement qu'implique l'expérience touristique conduit en principe à des « découvertes »;

- partir en voyage, c'est aussi opter pour la «liberté »;

- l'expérience touristique permet (en principe) de se «retrouver soi-même ».

\section{La rupture}

La rupture réfère au désir de se déconnecter, de se détendre et d'échapper au rythme de la vie quotidienne. Krippendorf (1987) parle de l'espace touristique comme de l'«antiquotidien», celui qui permet de fuir ${ }^{6}$ la monotonie de la vie courante, du travail, des rapports humains pauvres, etc., voire une existence qui engendre stress, épuisement physique et mental, vide intérieur et ennui. Par le voyage touristique, les gens cherchent un répit du fardeau des conditions habituelles ou des difficultés quotidiennes. C'est aussi une façon de remettre en cause les soucis quotidiens en prenant le temps de réfléchir, de puiser des 
forces et de retrouver une volonté de réagir différemment au retour. Le tourisme est donc un moment pour "faire le vide " pendant quelque temps pour "faire le plein" pour l'année (Gaudreau et al. 1999: 35). Cela est d'autant plus vrai dans le cas de populations marginalisées où le loisir touristique devient un temps de soulagement et d'espoir.

L'espace du voyage se compare donc à un " espace thérapeutique » qui offre détente et divertissements (cyclotourisme, activité de marche en plein air, visite de sites, etc.), lesquels ont des effets sur la bonne forme physique et mentale et sur l'équilibre psychologique et spirituel. Mais la rupture touristique a aussi comme fonction sociale de permettre à l'individu d'être "comme tout le monde" [émancipation], d'éviter la marginalisation [insertion] et d'avoir la possibilité de vivre des plaisirs et de nouvelles expériences qui s'exprimeront en souvenirs heureux à raconter [participation] (Conseil national du tourisme, 1998 ; Gaudreau et al., 1999).

\section{La découverte}

Par le changement de lieu et la rencontre avec l'Ailleurs, le touriste découvre et s'ouvre à "d'autres choses»: mentalités, paysages, modes de vie, odeurs/goûts, activités, etc. Le tourisme a donc une fonction de développement (intellectuel, physique) et d'enrichissement personnels et agit sur la compréhension des choses, les sensibilités et la spiritualité. Un enrichissement qui ne se réalise pas que durant le temps du séjour, mais aussi pendant l'«avant-voyage », par la recherche d'information, et l' " après-voyage ", par des actes de ressouvenance (regarder les photos, lire les documents acquis sur place).

L'Homme, à travers le touriste, prend donc conscience de la diversité du monde qui l'entoure et expérimente de nouvelles activités. C'est le cas, notamment, de tous ces jeunes qui font un séjour dans les camps de vacances durant l'été et reviennent fiers d'avoir taquiné la truite ou réussi leur brevet en canot (Touzin, 2005) ou encore des familles qui sont exposées, pendant leur séjour en camp, à d'autres modèles familiaux qu'ils peuvent reproduire par la suite à la maison pour améliorer leur vie familiale, tandis que les expériences de voyage à l'étranger sont sources de découvertes culturelles et de réflexion existentielle, spécialement dans le cas d'un voyage Nord/Sud.

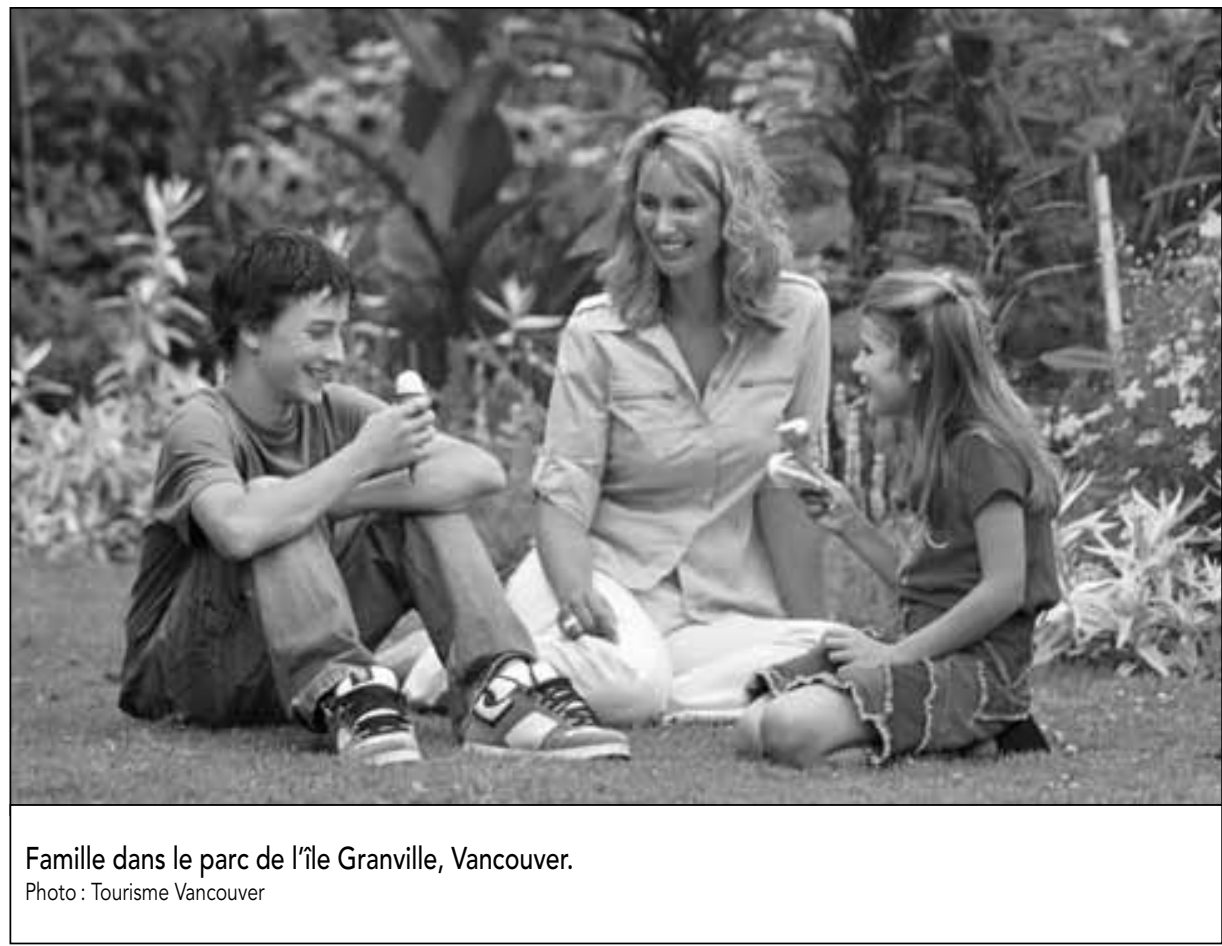

Le fait d'être témoin, oculaire et/ou auriculaire, d'une autre réalité [...], conscientise le touriste aux inégalités et aux injustices au niveau de la richesse et de l'accès aux ressources et aux services dits "essentiels». [...] II permet aussi de saisir la valeur des choses, comme les ressources naturelles (spécialement le prix de l'eau), de vieilles chaussures, un simple crayon, etc. (Proulx, 2005).

Ces nouveaux éléments de connaissance qu'apporte le tourisme conduisent nécessairement à une autre vision de la vie ou à un autre niveau d'analyse de la réalité et sont utiles au développement équilibré de l'individu et de sa personnalité, voire de son émancipation.

\section{La liberté}

Cette notion de liberté réfère à la possibilité de vivre autrement, d'une nouvelle manière d'être pour un temps limité, celui du séjour touristique. Laplante (1997) parle de vie jouée, d'un moment de plaisir et de défoulement où l'on se sent libre comme l'air. Cette liberté dispose, d'une part, à s'impliquer personnellement (et non simplement à assister) et, d'autre part, à réaliser des actions étrangères à son cadre de vie habituelle. On ira faire du rabaska, de l'escalade ou même on osera faire du paraski. On se sent rajeuni et plein de vitalité, c'est la «transe touristique », celle animée par un esprit festif et lu- dique. Le jeu permet à l'individu de s'extérioriser ou de jouer pour un instant à "être un autre », celui qu'il rêve d'être - le chef d'équipe plutôt que le timide ou l'adulte qui joue comme l'enfant. Ce grand jeu peut avoir un effet positif sur l'image et l'estime de soi.

C'est également le jeu qui facilite et favorise les relations sociales. Effectivement, la rencontre, la communication, l'intégration sociale sont particulièrement importantes dans cet " antiquotidien ludique ». Ainsi, durant ses vacances, l'individu redécouvre les échanges et établit plus aisément des contacts avec les autres. Le premier effet de cette socialisation se fait surtout sentir à l'intérieur de la famille, notamment parce que l'on part rarement seul mais avec sa "bulle sociale » (conjoint, famille, parents) (Laplante, 1997). Selon Gaudreau et al. (1999) un séjour de vacances familiales à l'extérieur du domicile a une incidence positive sur la relation parents-enfants, la communication et l'écoute envers l'enfant, la vie de couple, l'exercice de la discipline par les parents. Ainsi, cette période de détente et de divertissement que suppose le tourisme permet de s'accepter, de s'écouter, de dialoguer, de partager des activités, voire de consolider le lien familial (Conseil national du tourisme, 1998). Toutefois, les jeux de sociabilité ne se limitent pas à la bulle familiale; des échanges et des solidarités sont possibles avec les 
autres vacanciers et avec les « visités ». On parle alors des vacances comme d'un moment de mixité sociale, de brassage des populations et de cohésion sociale.Toujours selon le Conseil national du tourisme (1998: 70), "L'inclusion dans cet univers social différent crée une richesse qui permet l'accession à de nouvelles valeurs et à un comportement plus ouvert. Les enfants, les adolescents de tous âges, les adultes sortent de leur enfermement, s'expriment, se situent dans des groupes pluralistes et s'intègrent dans la vie collective " (Idem : 70), d'où l'idée que le tourisme est un outil dans un processus de réinsertion sociale.

\section{Se retrouver soi-même}

Le tourisme invite à réfléchir sur son identité, c'est-à-dire à se découvrir soi-même et à s'accepter, à accepter la différence et à affirmer la sienne. Cet exercice spirituel est possible par la rencontre et le regard que l'on porte sur l'Autre. "Les voyages à l'étranger ne nous apprennent pas seulement à distinguer entre les objets d'art, les coutumes ou les langues appartenant à des civilisations différentes. Ils ont aussi pour fonction de nous réconcilier avec nos valeurs sociétales et de nous conforter dans nos croyances et nos convictions collectives» (Amirou, 1995: 216). C'est par la comparaison que le touriste affirme son identité et sa distinction. "I/s aident et respectent les personnes âgées... nous on a pas le même attachement. " "lls ont des qualités humaines... nous on ne les exprime pas comme eux. » «I/s se contentent de peu pour être heureux... nous on a besoin de beaucoup de matériel " (Proulx, 2005). La rencontre touristique peut amener le touriste à envisager l'existence autrement et à faire davantage preuve de solidarité.

Également, l'activité touristique fait découvrir en nous de nouvelles capacités: nager, faire de l'escalade, etc. Elle nous éveille à de nouvelles sensibilités et émotivités face au paysage ou aux peuples qui nous accueillent et peut être révélatrice de nos goûts. Autrement dit, le voyage nous apprend à mieux nous connaître et à reconnaître nos propres habiletés. II est aussi l'occasion d'acquérir de nouvelles compétences et le moment idéal de braver nos peurs à un moment où nous sommes stimulés par l'esprit de liberté.

Tableau 1

Parallèle entre les bienfaits du loisir et les spécificités du tourisme

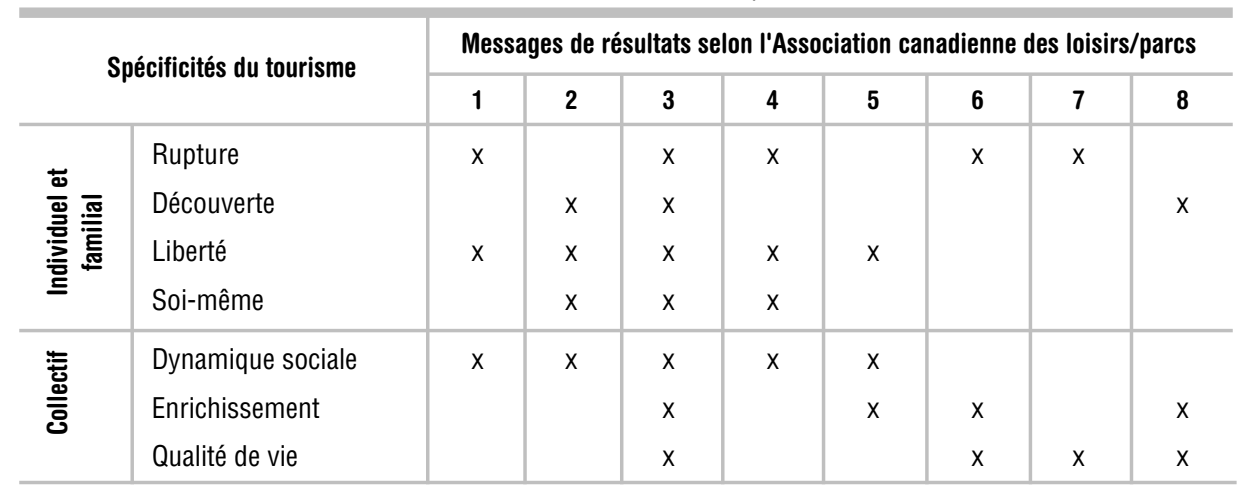

Source : Données recueillies par l'auteur.

\section{Le plan collectif}

L'espace touristique correspond aussi au terrain de jeu d'une communauté locale, lieu où la population locale participe à des loisirs. En termes de bien-être collectif, le tourisme est vu comme une solution, parmi d'autres, aux problèmes sociaux auxquels fait face une communauté (exclusion et isolement sociale, nouvelles formes de pauvreté, familles désœuvrées, etc.), en agissant comme outil de développement social. Effectivement, il favorise le développement d'une dynamique sociale et contribue à l'enrichissement et à la qualité de vie des collectivités (Proulx, 2005). Bref, le tourisme encourage la participation et la mobilisation de la communauté dans des projets collectifs. Pensons à la collaboration des citoyens dans les festivals par le bénévolat. Cette participation a une incidence sur la réinsertion sociale, le développement d'un sentiment d'appartenance et d'identification de la population à son territoire, la création de nouvelles solidarités et la cohésion sociale. Rappelons que l'implication bénévole influe également sur l'estime de soi des individus, la formation personnelle, la créativité, les compétences relationnelles, etc. C'est le pouvoir de la participation, de l'intégration et de l'émancipation individuelle dans le bien-être collectif. Le tourisme est aussi responsable d'un certain enrichissement collectif parce qu'il suscite une prise de conscience des richesses du milieu, une démocratisation de la culture (grâce au développement de produits culturels pour le tourisme), la création d'emplois et le développement de compétences locales. Finalement, par la préservation des milieux naturels et culturels qu'il engendre et l'aménagement d'infrastructures de loisir (pistes cyclables, sentiers de randonnée) qu'il oblige, il contribue à un environnement et à une qualité de vie meilleurs.

\section{Bienfaits du loisir touristique}

Cette brève analyse des effets individuels et sociaux du tourisme nous permet de constater qu'ils trouvent écho dans les huit messages de l'Association canadienne des loisirs/parcs cités précédemment. Le tableau 1 trace le parallèle entre ces messages et les spécificités du tourisme.

Nous constatons que c'est l'amélioration de la qualité de vie personnelle et sociale (troisième message) que le tourisme influe de façon prédominante, notamment parce que cette activité de loisir fournit une expérience globale qui touche la santé, le développement cognitif et moral et la sociabilité. Une expérience possible par cette impression « d'être » que permet l'« antiquotidien » durant un moment. L'influence positive du tourisme sur l'estime de soi (message 2) et sur le développement de comportements sociaux (message 3) est également à mentionner.

Le tourisme est donc loin de la seule récompense individuelle à un temps de travail durement accompli tout au long de l'année, voire un temps de ressourcement. On doit lui reconnaître aussi une valeur récréative, thérapeutique, émancipatrice et sociale.

\section{Conclusion}

La santé et le bien-être sont des préoccupations très présentes dans notre société actuelle. L'individu postmoderne recherche une meilleure qualité de vie, il s'occupe de sa santé, de celle de son corps et de son esprit. Les vacances et le départ touristique font partie des besoins qu'il doit satisfaire pour voir à sa santé et à son bien-être. 
Ces besoins sont justifiés à la lumière des nombreux bienfaits du tourisme, comme activité de loisir, sur les individus et les collectivités. Des bienfaits qui se situent sur le plan de la santé physique et mentale en général, mais aussi sur le plan de la spiritualité, du développement de la personne et de son identité, de la socialisation et de la cohésion sociale. Des bienfaits qui s'inscrivent parfaitement dans la définition du bienêtre que propose Breda (1998). Le tourisme contribue à la participation des individus à la vie sociale, à sa culture et à ses valeurs et permet le développement d'une personnalité, notamment par la découverte, la liberté et le retour à soi-même qu'il comporte.

II faut aussi admettre la contribution du tourisme au développement social des collectivités par l'aménagement d'infrastructures qu'il oblige et par la capacité de mobiliser les gens autour d'un projet collectif, de les intégrer socialement et de créer une solidarité et une fierté identitaire.

De tels bienfaits justifient que l'on reconnaisse le tourisme comme un droit pour tous et que l'on encourage son accessibilité. Malheureusement, encore aujourd'hui, près de $50 \%$ de la population des pays industrialisés (environ $45 \%$ des Québécois selon une enquête réalisée en $2003^{7}$ ) ne part pas en vacances (Proulx, 2004). Le manque d'argent et le manque de temps, notamment en raison d'une surcharge de travail, en sont les principales raisons. D'où l'importance d'accroître l'aide à l'accessibilité au tourisme qui passe principalement par un support des élus, voire par la mise en place d'une politique sociale du tourisme et par un appui significatif et récurrent aux organismes touristiques de la société civile, car ils reconnaissent déjà les fonctions humaines et sociales du tourisme.

En conclusion, les premiers touristes du $\mathrm{XVIII}$ siècle, ces aristocrates en mal du quotidien, avaient probablement déjà saisi l'essence du tourisme, celle de la détente, du divertissement, de la découverte, de la socialisation, de la thérapeutique et de la symbolique que procurent la rupture et la liberté.

Luce Proulx est chercheur-consultante en gestion et planification du tourisme et chargée de cours au Département d'études urbaines et touristiques de I'UQAM.

\section{Notes}

1 L'attrait de la campagne et de la villégiature débute dans la deuxième moitié du XVIIII siècle, mais s'intensifie surtout au XIX ${ }^{\ominus}$ siècle, pour ralentir vers la fin du XX.

2 L'idée du bonheur individuel est nouvelle et apparaît à l'époque du Romantisme. Cette époque est marquée d'ailleurs par des valeurs de bien-être, de libre expression de la sensibilité et d'évasion. Dans ce courant de pensée, on porte un nouveau regard à la Nature qui est dorénavant considérée, par les aristocrates, pour la valeur esthétique de son paysage, en plus de sa valeur thérapeutique. La campagne devient donc un espace de bonheur.

3 Le loisir est reconnu comme un droit dans divers documents officiels : Article 24 de la Déclaration universelle des droits de l'homme; Article 7 du Pacte international relatif aux droits économiques, sociaux et culturels; Article 1 de la Charte internationale pour le loisir ; Article 2 de la Loi canadienne sur les droits de la personne; Article 7 du Code mondial d'éthique du tourisme; et Déclaration nationale sur les loisirs de 1987.

4 Définition tirée du site Internet de l'Organisation mondiale de la Santé, [www.who.int/about/ definition/fr/print.html], une définition, dit-on, qui n'a pas été modifiée depuis 1946.

5 Le Petit Larousse Grand Format (2000) : «Fait d'être bien, satisfait dans ses besoins, ou exempt de besoins, d'inquiétudes ; sentiment agréable qui en résulte. " Le Multi Dictionnaire de la langue française (2003) : "Sentiment de bonheur et de sérénité. " Le Petit Robert électronique (nouvelle édition 2001) : "Sensation agréable procurée par la satisfaction de besoins physiques, l'absence de tensions psychologiques. »

6 Cette notion de fuite rappelle les motifs de départ des premiers touristes, ces aristocrates qui cherchaient aussi à fuir un quotidien dans lequel ils étaient inconfortables. La fuite serait-elle le fondement du tourisme?

7 Cette enquête a été réalisée par Léger Marketing dans le cadre d'une étude Omnibus, pour le compte de la Société des attractions touristiques du Québec, en collaboration avec Festivals et événements Québec, le Conseil québécois du loisir et l'ARUC (Alliance de recherche universités-communautés) en économie sociale via le Chantier d'activités partenariales Loisir et tourisme social. Elle concerne les comportements touristiques des Québécois à l'intérieur de leur province, de novembre 2002 à octobre 2003. Quelques résultats peuvent être consultés dans l'ouvrage de Proulx (2004).

\section{Bibliographie}

Amirou, Rachid (1995), Imaginaire touristique et sociabilités du voyage, Paris, Presses universitaires de France, $281 \mathrm{p}$.

Association canadienne des loisirs/parcs (1998), Le catalogue des bienfaits, Gloucester, Ontario, $246 \mathrm{p}$.
Boyer, Marc (1996), L'invention du tourisme, Paris, Gallimard, $153 \mathrm{p}$.

Breda, Jef (1998), La construction d'une mesure synthétique du bien-être, Recueil des communications du colloque Connaitre et surveiller pour agir sur la santé des populations, Les $4^{e}$ Rencontres du Réseau francophone international pour la promotion de la santé (RÉFIPS), Montréal, [www.refips.org/4er_rencontres].

Conseil de la santé et du bien-être (1998), Forum sur le développement social, Rapport remis au ministre de la Santé et des Services sociaux. Québec, 140 p.

Conseil national du tourisme, Section des Affaires Sociales (1998), Les vacances facteur d'insertion sociale, Paris, $125 \mathrm{p}$.

Conseil québécois du loisir CQL (2000), L'impact déterminant du loisir sur l'état de santé de la population, Avis du Conseil québécois du loisir à la Commission d'étude sur les services de santé et les services sociaux, Montréal, 31 p.

Conseil québécois du loisir (CQL) (2003), Le Conseil québécois du loisir: un réseau de réseaux, Planification stratégique 2003-2008, Montréal, $48 \mathrm{p}$.

Fréchette, Lucie (2000), À propos de la spécificité des Centres communautaires de loisir, Rapport présenté à la direction du loisir, Secrétariat au loisir et au sport, Ministère de la Santé et des Services sociaux du Québec, Hull, Géris-UQAH, 51 p.

Gaudreau, Louise, Louis Jolin, et Geneviève Buissonnet-Verger (1999), L'impact sur la vie familiale, Action communautaire réalisée au sein du Mouvement québécois des camps familiaux, Rapport d'évaluation, Montréal, Université du Québec à Montréal, Service aux collectivités, document $n^{\circ} 84,58 p$.

Groupe Confluences (1996), Invitation au voyage. Chances et limites du tourisme, Paris, Desclée de Brouwer, $147 \mathrm{p}$.

Krippendorf, Jost (1987), Les vacances, et après ? Pour une nouvelle compréhension des loisirs et des voyages, Paris, L'Harmattan, $239 \mathrm{p}$.

Laplante, Marc (1997), L'expérience touristique contemporaine. Fondements sociaux et culturels, Ste-Foy, Presses de I'Université du Québec, $189 \mathrm{p}$.

Proulx, Luce (2004), Pour un tourisme accessible à tous durable et solidaire, Montréal, Université du Québec à Chicoutimi, Cahiers de l'ARUC-ÉS, Collection Transfert, nº T-05-2004, 72 p.

Proulx, Luce (à paraître printemps 2006), « Impacts sociaux et culturels du tourisme : l'écotourisme une avenue à explorer ", dans Christiane Gagnon et Serge Gagnon (dir.), L'écotourisme: une innovation durable pour le développement viable des communautés locales? Presses de l'Université du Québec.

Sue, Roger (1980), Le loisir, Paris, Presses universitaires de France, Collection Que sais-je?, $128 \mathrm{p}$.

Touzin, Caroline (2005), « Camp de vacances : le retour ", La Presse, Montréal, samedi 9 juillet, p. A19. 